\title{
Hepatic fibrosis and short-term clinical efficacy after hepatic artery embolization for unresectable hepatocellular carcinoma using doxorubicin-eluting HepaSphere
}

\author{
Meng Chen", Rongde Xu\#, Xiaoming Chen, Qicong Mai \\ Department of Interventional, Guangdong Provincial People's Hospital/Guangdong Academy of Medical Sciences, Guangzhou 510030, China \\ Contributions: (I) Conception and design: M Chen, R Xu, X Chen; (II) Administrative support: None; (III) Provision of study materials or patients: \\ None; (IV) Collection and assembly of data: None; (V) Data analysis and interpretation: M Chen, R Xu, X Chen; (VI) Manuscript writing: All \\ authors; (VII) Final approval of manuscript: All authors. \\ "These authors contributed equally to this work. \\ Correspondence to: Xiaoming Chen. Department of Interventional, Guangdong Provincial People's Hospital/Guangdong Academy of Medical \\ Sciences, 12/f, Weilun Building, No. 2 Zhongshan Road, Yuexiu District, Guangzhou 510030, China. Email: chen.xiaoming@yandex.com.
}

\begin{abstract}
Background: Hepatocellular carcinoma (HCC) is most common malignancies around world. Transcatheter arterial chemoembolization (TACE) is recognized as the first-line treatment for HCC by NCCN, and its efficacy is widely reported. However, repeated TACE induces hepatic fibrosis. How to reduce hepatic fibrosis and retard cirrhosis is an urgent problem in treatment of HCC. To verify the efficacy of doxorubicin-eluting HepaSphere for TACE in the treatment of unresectable HCC.

Methods: We retrospectively analyzed 91 patients with unresectable HCC underwent TACE from June 2015 to June 2018. Among which, 51 cases were treated with HepaSphere-and 40 cases were treated with iodized oil. The primary endpoint was got according to the Modified Response Evaluation Criteria in Solid Tumors (mRECIST). Type IV collagen (IV-C), layer mucin (LN), amino-terminal propeptide of type III procollagen (PIIINP), and hyaluronic acid (HA) were tested before and after TACE treatment.

Results: Serologic factors of the groups were re-examined 3 days after TACE, which showed higher ALT and AST in the conventional TACE group than in the HepaSphere-TACE group $(\mathrm{P}<0.05)$. The postoperative efficacies were evaluated according to the mRECIST criteria. No difference in the short-term efficacy between these two groups $(\mathrm{P}>0.05)$ were found. Moreover, serologic factors for fibrosis were further re-examined 6 months later, showing no differences for IV-C and PIIINP (P=0.906 and 0.574, respectively). However, LN and HA were slightly higher in C-TACE group than HepaSphere-TACE group ( $\mathrm{P}=0.045$ and 0.048 , respectively).
\end{abstract}

Conclusions: HepaSphere-TACE is prevents the occurrence of late hepatic fibrosis effectively.

Keywords: Hepatocellular carcinoma (HCC); HepaSphere; iodized oil; hepatic fibrosis; hepatic cirrhosis; transarterial chemoembolization

Submitted Oct 07, 2019. Accepted for publication Dec 26, 2019.

doi: $10.21037 /$ tcr.2020.01.15

View this article at: http://dx.doi.org/10.21037/tcr.2020.01.15

\section{Introduction}

Hepatocellular carcinoma (HCC) is one of the most common malignancies in the world, and its mortality has risen to second place in cancer-related deaths $(1,2)$. About 800,000 patients die each year from HCC, and more than
$50 \%$ of new or fatal cases occur in China (3). Due to the imperceptible early symptoms, most patients have lost the optimal time for radical ablation or surgical resection at the time of diagnosis $(1,3)$. According to the NCCN guidelines, transcatheter arterial chemoembolization (TACE) is the 
first-line treatment for patients with advanced HCC, and its efficacy has been widely recognized.

Conventional transcatheter arterial chemoembolization (C-TACE) is a mixed emulsion of iodized oil and chemical agents, which could embolize the tumor supplying artery and cause hypoxia and local high concentrations of chemotherapeutic agents to induce tumor necrosis $(4,5)$. However, patients with HCC often need to undergo repeated TACE treatment, in which the hepatic fibrosis may be induced by the repeated lipiodol embolization (6). Cirrhosis is the last stage of hepatic fibrosis and it is one of the independent predictors of long-term efficacy for HCC (7). How to reduce hepatic fibrosis and slow the progression of cirrhosis is still an urgent problem to be solved in the comprehensive diagnosis and treatment of liver cancers.

Here, in this study, we included 91 cases of HCC that were treated with doxorubicin-loaded HepaSphere and traditional iodized oil-loaded drugs, respectively. The shortterm efficacy and postoperative hepatic fibrosis between these two groups were analyzed. The results indicated that doxorubicin-loaded HepaSphere might reduce hepatic fibrosis after repeated TACE.

\section{Methods}

\section{Study design}

This single-center retrospective study was conducted at the Guangdong Provincial People's Hospital. The study has been approved by the Ethics Committee of the Guangdong Provincial People's Hospital, and all patients or their families have signed relevant informed consent. The number of the ethical approval is No. GDREC 2016437H. All surgeries were performed by a surgeon with more than 20 years of treatment experience, and postoperative efficacy evaluation was performed by two or more senior clinicians.

\section{Inclusion and exclusion criteria}

Before being included in the analysis, patients must meet all the following criteria: (I) age $>18$ years, the patient was diagnosed as HCC according to the histological examination or imaging criteria of the European Association of Liver Research (EASL); (II) tumor diameter $\leq 150 \mathrm{~mm}$, Child-Pugh of Liver function $\leq 7$ points, Eastern Cancer Cooperative Group (ECOG) score: 0-2 points; (III) patients were BCLC stage B or BCLC stage A without radical treatment and the liver function Child-Pugh $\leq 7$ points, patients were BCLC C stage, ECOG $\leq 2$, and the expected survival period $\geq 6$ months.

Patients was excluded from the study if any of the following criteria were met: (I) presence of clinically serious vascular diseases such as cerebrovascular accident (within 6 months prior to TACE), myocardial infarction (within 6 months prior to TACE), uncontrollable hypertension after appropriate medical treatment, unstable angina pectoris, congestive heart failure (NYHA class 2-4), arrhythmia requiring medication; (II) allergic to pirarubicin or contrast media; (III) pregnant or lactating women; (IV) patients with other concomitant malignancies.

\section{Procedure of hepatic artery embolization}

A venous access was established on the day of surgery. In addition, the patients were given antiemetic (Dolasetron, $25 \mathrm{mg}$, IV, Haisco Pharmaceutical Group, Tibet, China) and antibiotic (Cefazoline, $1 \mathrm{~g}$, IV, China Resources Group, Beijing, China). Surgery was performed under local anesthesia (or general anesthesia if necessary). Seldinger technology was used for femoral artery puncture. RH or YASHIRO catheters (TERUMO, Tokyo, Japan) were used for hepatic artery angiography, and Finecross microcatheters (TERUMO, Tokyo, Japan) were used to select HCCfed arteries. The drug-loaded microsphere suspension/ iodinated oil emulsion was injected in a pulsed manner to embolize the tumor supplying artery. The injection rate was $1 \mathrm{~mL}$ per minute until the end of embolization was reached (tumor blood flow slowed down and the contrast agent disappeared after 2-5 heartbeats). For tumors involving 2 hepatic lobes, the more severe hepatic lobe was treated first, then the contralateral lobe was treated after a 2-4-week interval, and liver function must be restored to baseline levels prior to treatment.

In the HepaSphere-TACE group, all patients underwent TACE surgery using 50-100 $\mu \mathrm{m}$ HepaSphere microspheres (Merit Medical, USA). Pirarubicin was loaded using a two-step technique and the embolization procedure was shown in Figure 1. In the C-TACE group, all patients were treated with iodized oil (Guerbet, France) injection as an embolic material. The dosage of iodized oil was determined according to the size and vascular supply of the tumor, but the maximum dose did not exceed $20 \mathrm{~mL}$. Pirarubicin $(50 \mathrm{mg})$, iodized oil and a nonionic contrast agent (1:1) were emulsified. If the end of embolization was not reached after all of the iodized oil emulsion had been injected, appropriate doses of 100-300, 300-500, and 500-700 $\mathrm{mm}$ 


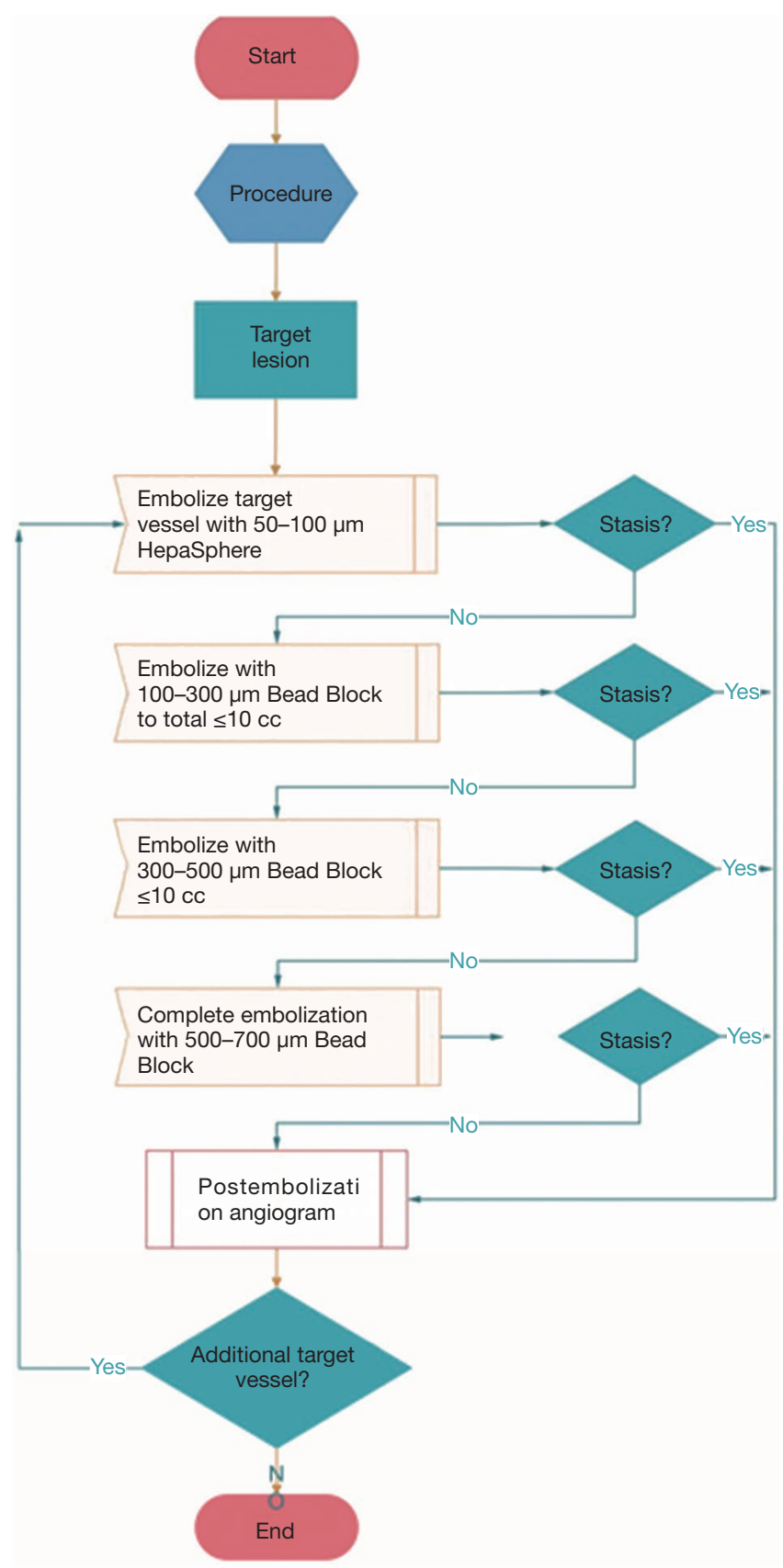

Figure 1 Procedure of HepaSphere-TACE for HCC. In the HepaSphere-TACE group, Pirarubicin $50 \mathrm{mg}$ was loaded onto HepaSphere (25 mg). The nutrient vessel of tumor was embolized using HepaSphere microspheres loaded with pirarubicin. After all the microspheres were injected, added Bead Block of appropriate specifications necessary to achieve stasis, depending on assessment of a combination of tumor volume and vascularity. Stasis was defined as the absence of antegrade flow within a vessel such that contrast filling the target vessel persisted, without washout, five cardiac beats after the injection of contrast. blank microspheres were administered to embolize the blood supply to the tumor until the end reached. Hepatic fibrosis (IV-C, LN, PIIINP and HA) were determined using chemiluminescence immunoassay. All kits were purchased from Sigma, USA.

Peripheral venous blood was collected from all patients three days before and after the surgery for the corresponding blood test. The decision regarding retreatment or not was based on enhanced CT/MR obtained every other month. And the internationally recognized solid tumor improvement response assessment (mRECIST) was used for efficacy evaluation. Patients with CR (complete response) entered the follow-up system. In the case of PR (partial response) or SD (stable disease), repeated treatment was considered, but a 4-6-week interval from the last treatment was required, and liver function must be restored to baseline before the repeated treatment. If PD (progressive disease) persisted after TACE treatment for the same lesion twice, patients should no longer receive TACE. Changes of liver function were compared at 3 days and 1 month before and after the treatment, and the treatment effects were evaluated at 1,3, and 6 months later, respectively. The severity of hepatic fibrosis was compared within 6 months after treatment between the two groups.

\section{Statistical analysis}

Data analysis was performed using SPSS 20.0 statistical analysis software (IBM, Armonk, New York, USA). Data are expressed as mean $\pm \mathrm{SD}$. Inter-group comparisons were performed using independent sample t-tests. The calculated data were described using the number of cases or percentages and compared using the $\chi^{2}$-test. The test level was set to $\alpha=0.05$, and a $\mathrm{P}$ value $<0.05$ indicated statistical significance.

\section{Results}

\section{Baseline of the patients}

We included a total of 91 patients with advanced liver cancer who were treated in this hospital between May 2015 and May 2018 in this study. These patients were divided into groups as follows: 51 patients in trial group receiving HepaSphere-TACE and 40 patients in control group receiving C-TACE. The details were shown in Table 1. There were no significant differences in age, gender constituent ratio, histories of hepatitis, drinking or Hepatic 
Table 1 The physiological baseline characteristics of the study cohort

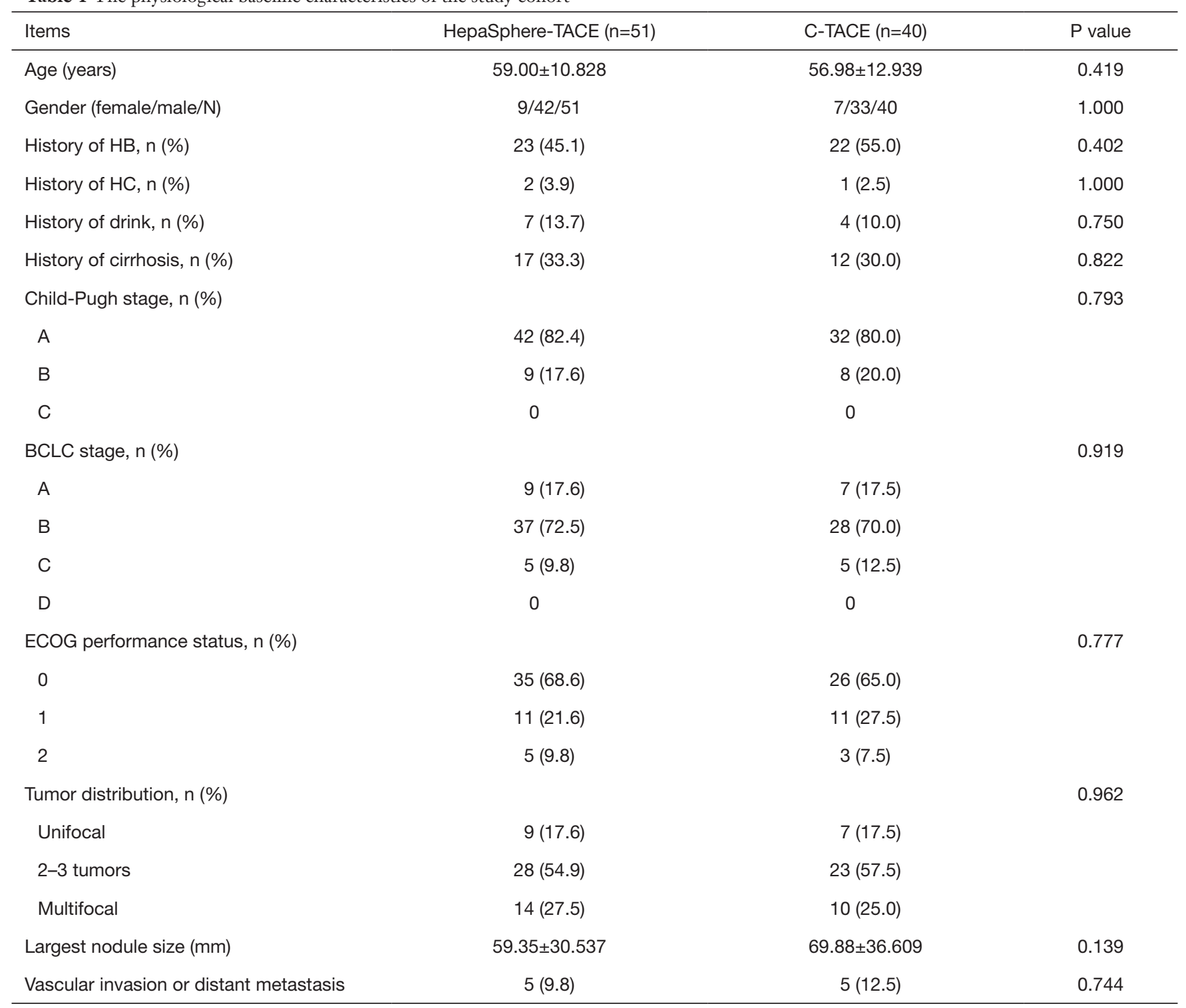

Prior therapy includes surgical or medical therapy not directed toward current tumor burden or systemic therapy. BCLC, Barcelona Clinic Liver Cancer; ECOG, Eastern Cooperative Oncology Group.

cirrhosis, liver function Child-Pugh scoring, BCLC staging, ECOG staging, and tumor condition between the two groups (all $\mathrm{P}$ value $>0.05$ ).

Peripheral venous blood was collected from all included cases within 3 days before surgery for the determination of corresponding factors (albumin, ALT, AST, total bilirubin, cholinesterase, prothrombin activity, platelets and alphafetoprotein) and serologic factors for hepatic fibrosis. None of the results showed any significant differences in the corresponding blood indexes between these two groups (all
$\mathrm{P}$ value $>0.05)$. See Tables 2 and 3 for details.

\section{Liver function after treatment}

Liver function and other serological factors were reexamined in all patients within 3 days after TACE treatment. The results showed changes in postoperative liver function in both groups. In the TACE treatment group, the changes in patients' albumin, total bilirubin, cholinesterase, prothrombin activity, platelet and alpha- 
Table 2 The serological baseline characteristics of the study cohort

\begin{tabular}{lccc}
\hline Items & HepaSphere-TACE $(\mathrm{n}=51)$ & C-TACE $(\mathrm{n}=40)$ & P value \\
\hline Albumin & $35.047 \pm 4.7322$ & $36.245 \pm 4.9931$ & 0.245 \\
ALT & $40.08 \pm 28.334$ & $46.40 \pm 52.698$ & 0.466 \\
AST & $53.90 \pm 38.139$ & $64.75 \pm 66.883$ & 0.332 \\
Total bilirubin & $21.645 \pm 11.5814$ & $19.015 \pm 10.5462$ & 0.267 \\
Cholinesterase & $5,458.86 \pm 1,823.515$ & $5801.45 \pm 2,106.660$ & 0.408 \\
Prothrombin activity & $89.45 \pm 14.492$ & $92.90 \pm 13.046$ & 0.242 \\
Platelet & $138.84 \pm 55.434$ & $169.28 \pm 96.212$ & 0.061 \\
Alpha-fetoprotein & $6,776.664 \pm 16,295.5952$ & $6,217.937 \pm 14,888.1539$ & 0.867 \\
\hline
\end{tabular}

ALT, alanine aminotransferase; AST, aspartate aminotransferase.

Table 3 The hepatic fibrosis baseline of the study cohort

\begin{tabular}{lccc}
\hline Parameters & HepaSphere-TACE $(\mathrm{n}=51)$ & C-TACE $(\mathrm{n}=40)$ & P value \\
\hline IV-C & $110.3714 \pm 74.37717$ & $131.5425 \pm 153.96306$ & 0.391 \\
LN & $64.1035 \pm 39.78016$ & $74.6697 \pm 61.97047$ & 0.327 \\
PIIINP & $12.3882 \pm 5.130032$ & $13.7607 \pm 7.21875$ & 0.292 \\
HA & $239.6320 \pm 305.93052$ & $208.8608 \pm 242.33391$ & 0.604 \\
\hline
\end{tabular}

IV-C, type IV collagen; LN, layer mucin; PIIINP, amino-terminal propeptide of type III procollagen; HA, hyaluronic acid.

fetoprotein were not significantly different from those before treatment $(\mathrm{P}>0.05)$. However, there was a significant difference $(\mathrm{P}<0.05)$ for alanine aminotransferase (ALT) and aspartate aminotransferase (AST) levels. Moreover, changes in ALT and AST were more significant in C-TACE group than in HepaSphere-TACE group (Table 4).

We also reviewed the relevant factors one month after the patient was treated. The results showed that albumin, ALT, AST, total bilirubin, cholinesterase, prothrombin activity, platelet, alpha-fetoprotein and other factors were not significantly different $(\mathrm{P}>0.05$, Table 5$)$.

\section{Short-term efficacy of HepaSphere-TACE and C-TACE}

We used mRECIST to evaluate the treatment efficacy in surgery for both groups one, three and six months later (Figure 2), and used the $\chi^{2}$ test to analyze shortterm postoperative outcomes. The results showed that all $\mathrm{P}$ values were $>0.05$, indicating there was no significant difference in short-term efficacy between the HepaSphereTACE group and the C-TACE group (Table 6). The chisquare test was used for comparison between the two groups. $\mathrm{P}<0.05$ was considered significant. There was no difference between the first month, the third month, and the sixth month between the two groups (OR: objective response rate $=\mathrm{CR}+\mathrm{PR}, \mathrm{DC}$ : disease control rate $=\mathrm{CR}+$ $\mathrm{PR}+\mathrm{SD})$.

\section{Comparison of hepatic fibrosis changes in the two groups}

There were no significant differences in preoperative IVC, LN, PIIINP and HA between HepaSphere-TACE group and C-TACE group. After 6 months of continuous treatment, hepatic fibrosis was reviewed in both groups, showing no significant difference in CIV and PIIINP levels between the two groups $(\mathrm{P}=0.906,0.574$, respectively). However, LN and HA levels were slightly higher than in the C-TACE group than that of HepaSphere-TACE group $(\mathrm{P}=0.045$ and 0.048 , respectively) (Table 7).

\section{Discussion}

Compared to the traditional TACE technology, HepaSphere-TACE has the advantages of non-degradability 
Table 4 Comparison of liver function on the 3rd day after operation in both groups

\begin{tabular}{|c|c|c|c|}
\hline Parameters & HepaSphere-TACE $(n=51)$ & C-TACE $(n=40)$ & $P$ value \\
\hline ALT & $43.81 \pm 32.624$ & $71.78 \pm 87.484$ & 0.038 \\
\hline AST & $57.69 \pm 40.446$ & $101.28 \pm 101.161$ & 0.006 \\
\hline Total bilirubin & $24.113 \pm 14.6082$ & $26.305 \pm 29.0519$ & 0.640 \\
\hline Prothrombin activity & $88.41 \pm 15.326$ & $90.90 \pm 13.700$ & 0.423 \\
\hline Platelet & $156.75 \pm 167.664$ & $159.50 \pm 86.094$ & 0.925 \\
\hline Alpha-fetoprotein & $6,730.908 \pm 16,291.0440$ & $8,319.067 \pm 1,7305.4600$ & 0.654 \\
\hline
\end{tabular}

ALT, alanine aminotransferase; AST, aspartate aminotransferase.

Table 5 Comparison of liver function in 1 month after operation in both groups

\begin{tabular}{lccc}
\hline Parameters & HepaSphere-TACE $(\mathrm{n}=51)$ & C-TACE $(\mathrm{n}=40)$ & $\mathrm{P}$ value \\
\hline Albumin & $34.584 \pm 4.6584$ & $34.678 \pm 5.2043$ & 0.928 \\
ALT & $41.312 \pm 31.4322$ & $60.228 \pm 64.6390$ & 0.070 \\
AST & $76.882 \pm 122.9738$ & $113.475 \pm 154.0055$ & 0.211 \\
Total bilirubin & $30.584 \pm 52.3265$ & $26.452 \pm 26.5851$ & 0.650 \\
Cholinesterase & $5,268.82 \pm 2,198.602$ & $5,105.25 \pm 2,462.365$ & 0.739 \\
Prothrombin activity & $88.71 \pm 16.199$ & $87.25 \pm 15.400$ & 0.665 \\
Platelet & $138.39 \pm 64.772$ & $172.88 \pm 103.282$ & 0.055 \\
Alpha-fetoprotein & $7,067.283 \pm 16,734.9663$ & $9,964.659 \pm 19,047.7122$ & 0.443 \\
\hline
\end{tabular}

Comparison among two groups was performed by Student's $t$-test. $\mathrm{P}<0.05$ was considered significant. No difference of liver function index among two groups was shown.

of the embedded microspheres and sustained release of the drug. HepaSphere-TACE up-regulates the concentration of chemotherapeutic drugs at the site of tumor embolization under the premise of reliable embolization. In addition, according to meta-analysis, the side effects of HepaSphereTACE, including fever, fatigue, abdominal pain, nausea, alopecia, myelosuppression and other common adverse reactions, are less than traditional embolization treatments of iodized oil (8).

In this study, we found that patients with HepaSphereTACE and C-TACE treatments both induced reversible liver function changes, mainly in the reduction of albumin and cholinesterase, as well as increase of ALT and AST. However, after C-TACE treatment, patients with increased higher ALT and AST, which may be related to the rapid release of chemotherapy drugs caused by C-TACE.
However, drug-loaded microspheres currently used in clinical (such as HepaSphere and DC-bead) are mostly non-degradable microspheres (9). Therefore, there is an urgent demand to find new materials that are more susceptible to degradation than iodized oil. Otherwise, ectopic embolization of the microspheres would result in irreversible damage. In general, HepaSphere microspheres are TACE safe and effective embolic agents. Its effects on liver function and postoperative complications are within acceptable limits, but more attention should be paid to selecting the blood supply artery to avoid ectopic embolization.

Currently, researches about drug-loaded microspheres and traditional lipiodol have been reported. Although drugloaded microspheres are significantly superior to iodized oil in pharmacokinetics, there is no strong evidence for 


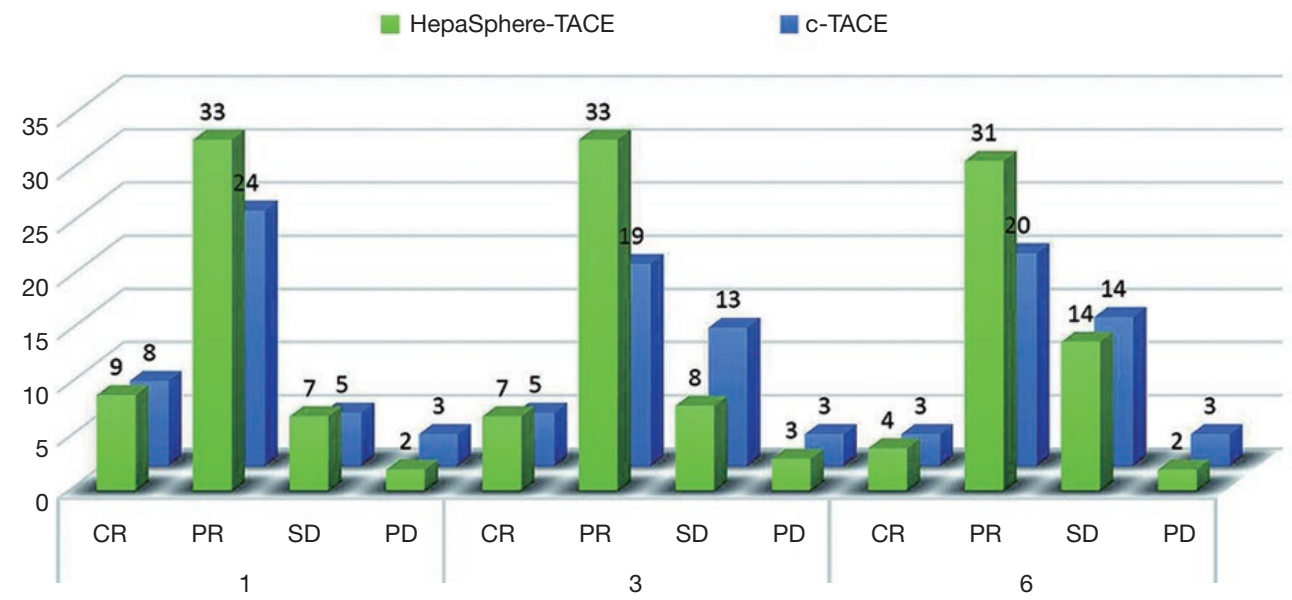

Figure 2 Comparison among two groups was performed by Chi-square test. $\mathrm{P}<0.05$ was considered statically significant. No differences were found in $1^{\text {st }}, 3^{\text {rd }}$ and $6^{\text {th }}$ months after treatment in both groups $(\mathrm{P}=0.875,0.259,0.704$, respectively).

Table 6 Comparison of recent treatment efficiency between the two groups

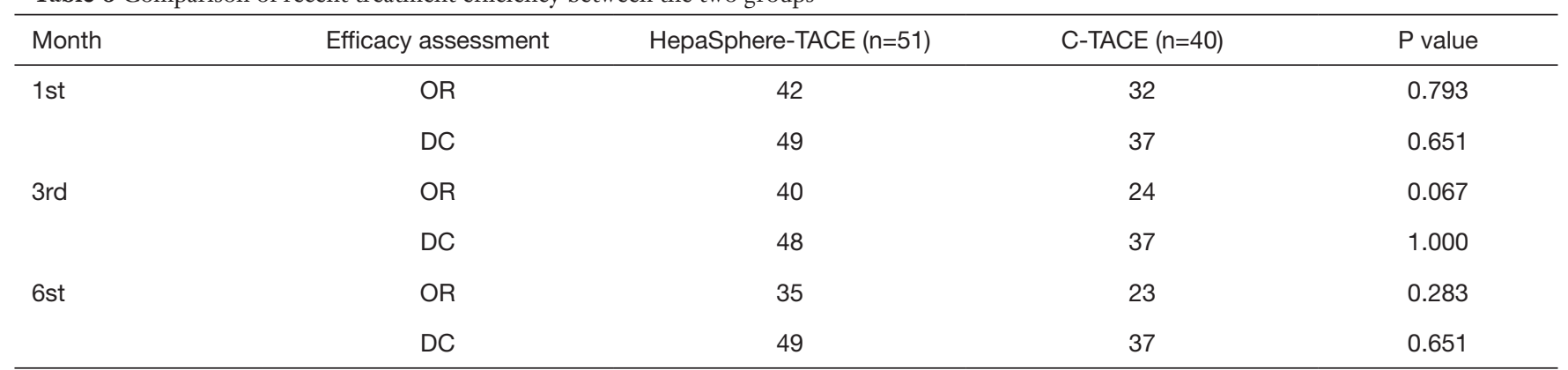

Comparison among two groups was performed by Chi-square test. $\mathrm{P}<0.05$ was considered significant. No difference in 1 st, 3 rd, 6 th month among two groups was shown. OR, objective response; DC, disease control.

Table 7 Comparison of liver fibrosis after 6 months in the two groups

\begin{tabular}{lccc}
\hline Parameters & HepaSphere-TACE $(\mathrm{n}=51)$ & C-TACE $(\mathrm{n}=40)$ & P value \\
\hline IV-C & $137.9990 \pm 111.31665$ & $140.8385 \pm 115.39194$ & 0.906 \\
LN & $77.3522 \pm 45.9516$ & $104.3360 \pm 79.19955$ & 0.045 \\
PIIINP & $15.9671 \pm 8.36818$ & $17.0202 \pm 9.38048$ & 0.574 \\
HA & $216.3737 \pm 238.43665$ & $339.4758 \pm 346.31562$ & 0.048 \\
\hline
\end{tabular}

IV-C, type IV collagen; LN, layer mucin; PIIINP, amino-terminal propeptide of type III procollagen; HA, hyaluronic acid.

the superiority of drug-loaded microspheres to iodized oil in HCC treatment. Here, we retrospectively analyzed 91 patients treated with different TACE (including 51 cases with HepaSphere-TACE, 40 cases with C-TACE). The results showed no significant difference in shortterm efficacy between the two methods at the first month, the third month, and the sixth month after treatment. In a meta-analysis of 1,449 patients, there was no significant difference in tumor response rate or survival between the DEB-TACE group and the C-TACE group (10). And in a comparative study of the efficacy of commonly used drugloaded microspheres, there was no significant difference in the short-term efficacy of DC-Bead TACE, HepaSphereTACE and C-TACE (11). In addition, in a prospective study 
conducted by Brown et al. (9), 101 patients with HCC were randomized to receive drug-loaded microspheres or blank microspheres as embolic material. The overall survival rates for the two groups were 20.8 and 19.6 months, respectively $(\mathrm{P}=0.64)$. However, in this study, 34 patients were BCLC patients with stage $\mathrm{C}, 60$ patients had multiple intrahepatic lesions, and 19 patients had portal vein tumor thrombus. According to NCCN guideline, the first-line therapeutic regimen for patients with BCLC stage $\mathrm{C}$ was targeted and drug-based comprehensive treatment (10), but all patients enrolled in this study received TACE, without mentioning essential basic targeted therapy, and the number of cases enrolled in this study was another limitation.

In addition, cirrhosis is one of the factors that affect the prognosis of liver cancer, and liver fibrosis is an important cause of cirrhosis. . Repeated TACE treatment may lead to hepatocyte damage and hepatic fibrosis in HCC patients, which would further impair liver function and lead to chronic decompensation of liver (10). Currently, the best method for clinically determining the severity of hepatic fibrosis is liver biopsy. However, due to its invasiveness and poor reproducibility, repeated biopsy monitoring during long-term treatment is difficult. "APASL consensus guideline: Invasive and non-invasive assessment of hepatic fibrosis: a 2016 update" (4) and "2015 EASL-ALEH clinical practice guideline: Non-invasive tests for evaluation of liver disease severity and prognosis" (12), serologic markers of hepatic fibrosis provide high-quality evidence in the diagnosis or exclusion of significant hepatic fibrosis and cirrhosis. Recent studies that have shown that compared with transient elastography (FibroScan), type IV collagen (IV-C), laminin (LN), type III procollagen $\mathrm{N}$-terminal peptide (PIIINP), serum hyaluronic acid (HA), alanine aminotransferase (ALT), aspartate aminotransferase (AST), and other hepatic fibrosis serological markers have equally good performance in predicting hepatic fibrosis (13). Thus, the combination of serologic markers for fibrosis test maybe a reliable non-invasive method for the prediction of hepatic fibrosis. In this study, we compared the changes in hepatic fibrosis markers before and after surgery in the two groups. The results showed no significant difference in IV-C and PIIINP levels between the two groups $(\mathrm{P}=0.906$ and 0.574 , respectively), but the $\mathrm{LN}$ and HA levels in the C-TACE group were slightly higher than those in the HepaSphereTACE group ( $\mathrm{P}=0.045$ and 0.048 , respectively). Therefore, repeated C-TACE surgery in HCC patients is more likely to increase liver fibrosis and affect liver reserve function. Inflammation occurs in all tissues in response to injury or stress, and it is also a key factor in hepatic fibrosis (14). At the same time, hepatic fibrosis represents the liver tissue wound repair process, which indirectly objectively reflects the severity of pathological damage to liver $(15,16)$. Highconcentration chemotherapeutic drugs in patients with HCC after treatment with TACE are the main cause of postoperative liver inflammation. Under the premise of ensuring the therapeutic effect, thus, how to reduce the concentration of chemotherapeutic drugs in normal liver is an effective measure to slow the progression of hepatic fibrosis. Kong et al applied low-dose and conventionaldose chemotherapy drugs to 50 patients with HCC during C-TACE. After C-TACE, four serological markers of hepatic fibrosis were significantly increased in the conventional dose group, while there was no significant difference in the low dose group (17). In another study, 36 patients with HCC who received TACE were included in low-dose $(n=15)$ and conventional-dose $(n=21)$ chemotherapeutic groups for analysis. In conventionaldose group, IV-C, LN, hPC-III (human pro-collagen typeIII), HA and transforming growth factor-beta 1 (TGF- $\beta 1$ ) significantly increased after surgery $(\mathrm{P}<0.01)$, while no significant differences were observed in low-dose group except for TGF- $\beta 1$ (18).

In a pharmacokinetic study of HepaSphere (19), 45 HCC patients received TACE using HepaSphere microspheres or iodized oil emulsions. The doxorubicin AUC of the HepaSphere-TACE group $(35,195 \pm 27,873 \mathrm{ng} \times \mathrm{min} / \mathrm{mL})$ was significantly lower than that of the conventional C-TACE group $(103,960 \pm 16,652 \mathrm{ng} \times \mathrm{min} / \mathrm{mL} ; \mathrm{P}=0.009)$. At the same time, the Cmax of the HepaSphere-TACE group $(83.9 \pm 32.1 \mathrm{ng} / \mathrm{mL})$ was also significantly lower than that of the C-TACE group $(761.3 \pm 58.8 \mathrm{ng} / \mathrm{mL} ; \mathrm{P}=0.002)$. HepaSphere may be one of the best embolic materials for TACE in the treatment of patients with Hepatic cirrhosis by its locally sustained release action and reliable embolization efficacy. However, regardless of HepaSphereTACE or C-TACE, embolization-induced hepatic hypoxia will inevitably aggravate the fibrosis process of liver tissues; accordingly, selecting tumor blood-feeding vessels as far as possible is an effective method to reduce normal liver injuries.

In summary, although there may not be any significant difference between the short-term and long-term effects of drug-loaded microspheres (such as HepaSphere and C-TACE), HepaSphere may have better application prospects in patients with liver cirrhosis compared to other drug-loaded microspheres. In addition, by repeatedly 
receiving C-TACE, DEB-TACE treatment may achieve better results in patients who have no response or even disease progression (20). In the embolization treatment of HCC, the relevant factors for drug-loaded microspheres have not been determined, and more relevant studies should be done to explore whether the efficacy is related to genotyping, tumor pathological type, and tumor anatomy, as well as HCC primary and metastasis.

\section{Acknowledgments}

Funding: None.

\section{Footnote}

Conflicts of Interest: All authors have completed the ICMJE uniform disclosure form (available at http://dx.doi. org/10.21037/tcr.2020.01.15). The authors have no conflicts of interest to declare.

Ethical Statement: The authors are accountable for all aspects of the work in ensuring that questions related to the accuracy or integrity of any part of the work are appropriately investigated and resolved. The study was conducted in accordance with the Declaration of Helsinki (as revised in 2013). The study has been approved by the Ethics Committee of the Guangdong Provincial People's Hospital, and all patients or their families have signed relevant informed consent. The number of the ethical approval is No. GDREC 2016437H.

Open Access Statement: This is an Open Access article distributed in accordance with the Creative Commons Attribution-NonCommercial-NoDerivs 4.0 International License (CC BY-NC-ND 4.0), which permits the noncommercial replication and distribution of the article with the strict proviso that no changes or edits are made and the original work is properly cited (including links to both the formal publication through the relevant DOI and the license). See: https://creativecommons.org/licenses/by-nc-nd/4.0/.

\section{References}

1. Ghouri YA, Mian I, Rowe JH. Review of hepatocellular carcinoma: Epidemiology, etiology, and carcinogenesis. J Carcinog 2017;16:1.

2. Maida $M$, Orlando $E$, Cammà $C$, et al. Staging systems of hepatocellular carcinoma: A review of literature. World J
Gastroenterol 2014;20:4141-50.

3. Feng RM, Zong YN, Cao SM, et al. Current cancer situation in China: good or bad news from the 2018 Global Cancer Statistics? Cancer Commun (Lond) 2019;39:22.

4. Wáng YX, De Baere T, Idée JM, et al. Transcatheter embolization therapy in liver cancer: an update of clinical evidences. Chin J Cancer Res 2015;27:96-121.

5. Lee KH, Liapi E, Vossen JA, et al. Distribution of Iron Oxide-containing Embosphere Particles after Transcatheter Arterial Embolization in an Animal Model of Liver Cancer: Evaluation with MR Imaging and Implication for Therapy. J Vasc Interv Radiol 2008;19:1490-6.

6. Wang $\mathrm{Z}$, Zhang $\mathrm{H}$, Zhao $\mathrm{H}$, et al. Repeated transcatheter arterial chemoembolization is safe for hepatocellular carcinoma in cirrhotic patients with transjugular intrahepatic portosystemic shunt. Diagn Interv Radiol 2014;20:487-91.

7. Saviano A, Baumert TF. Mortality from liver cirrhosis and HCC in the DAA era: success in viral control is darkened by raise of metabolic disease. Hepatobiliary Surg Nutr 2019;8:307-10.

8. Duan F, Wang EQ, Lam MGEH, et al. Super selective Chemoembolization of HCC Comparison of Shortterm Safety and Efficacy between Drug-eluting LC Beads, Quadra Spheres, and Conventional Ethiodized Oil Emulsion. Radiology 2016;278:612-21.

9. Brown KT, Do RK, Gonen MG, et al. Randomized Trial of Hepatic Artery Embolization for Hepatocellular Carcinoma Using Doxorubicin-Eluting Microspheres Compared With Embolization With Microspheres Alone. J Clin Oncol 2016;34:2046-53.

10. Benson AB, D'Angelica MI, Abbott DE, et al. NCCN Guidelines Insights: Hepatobiliary Cancers, Version 1.2017. J Natl Compr Canc Netw 2017;15:563-73.

11. Shiha G, Ibrahim A, Helmy A, et al. Asian-Pacific Association for the Study of the Liver (APASL) consensus guidelines on invasive and non-invasive assessment of hepatic fibrosis: a 2016 update. Hepatol Int 2017;11:1-30.

12. European Association for Study of Liver; Asociacion Latinoamericana para el Estudio del Higado. EASLALEH Clinical Practice Guidelines: Non-invasive tests for evaluation of liver disease severity and prognosis. J Hepatol 2015;63:237-64.

13. Dong $\mathrm{H}, \mathrm{Xu} \mathrm{C}, \mathrm{Zhou} \mathrm{W}$, et al. The combination of 5 serum markers compared to FibroScan to predict significant hepatic fibrosis in patients with chronic hepatitis B virus. Clin Chim Acta 2018;483:145-50. 
14. Nguyen-Lefebvre AT, Ajith A, Portik-Dobos V, et al. The innate immune receptor TREM-1 promotes liver injury and fibrosis. J Clin Invest 2018;128:4870-83.

15. Duran A, Hernandez ED, Reina-Campos M, et al. p62/ SQSTM1 by Binding to Vitamin D Receptor Inhibits Hepatic Stellate Cell Activity, Fibrosis, and Liver Cancer.\%A Duran A. Cancer Cell 2016;30:595-609.

16. Maretti-Mira AC, Wang X, Wang L, et al. Incomplete differentiation of engrafted bone marrow endothelial progenitor cells initiates hepatic fibrosis in the rat. Hepatology 2019;69:1259-72.

17. Kong WD, Cao JM, Xu J, et al. Impact of low versus conventional doses of chemotherapy during transcatheter arterial chemo-embolization on serum fibrosis indicators and survival of liver cancer patients. Asian Pac J Cancer

Cite this article as: Chen M, Xu R, Chen X, Mai Q. Hepatic fibrosis and short-term clinical efficacy after hepatic artery embolization for unresectable hepatocellular carcinoma using doxorubicin-eluting HepaSphere. Transl Cancer Res 2020;9(3):1361-1370. doi: 10.21037/tcr.2020.01.15
Prev 2012;13:4757-61.

18. Li H, Hu Y, Li N, et al. Hepatic fibrosis and five year survival of hepatocellular cancer cases undergoing transcatheter arterial chemo embolization using small doses. Asian Pac J Cancer Prev 2012;13:1589-93.

19. Malagari K, Pomoni M, Moschouris H, et al. Chemoembolization of hepatocellular carcinoma with HepaSphere 30-60 $\mu \mathrm{m}$. Safety and efficacy study. Cardiovasc Intervent Radiol 2014;37:165-75.

20. Song DS, Choi JY, Yoo SH, et al. DC Bead Transarterial Chemoembolization Is Effective in Hepatocellular Carcinoma Refractory to Conventional Transarteral Chemoembolization: A Pilot Study. Gut Liver $2013 ; 7: 89-95$. 